a function $q \in C[0, \infty)$ which does not vanish identically in the right neighbourhood of 0 such that $f=p_{1} / q$ and $g=p_{1} / q$, where $p_{1} p_{2} \epsilon O[0, \infty)$. Let $M_{q}$ be the set of all operators which can be represented in the form $p / q(p \in C[0, \infty))$. By Theorem $V, f$ can be approximated by sums $(2)$ in the topology of $M_{q}$. But every sequence which converges in the topology of $M_{q}$ also converges operationally, which proves our assertion. Taking in particular $g=1$, we see that every operator from $M_{+}$can be approximated by polynomials $\lambda_{1} h^{\tau_{1}}+\ldots+\lambda_{n} h^{\tau_{n}}$ of the shift-operator with positive $\tau_{i}$.

5. We have considered, so far, functions, distributions and operators defined on the one-dimensional real space $R$. However, all the theorems can also be interpreted in the Euclidean space $R^{m}$ of any number of dimensions. Then by an interval $[0, T]$ we understand the set of points $t=\left(t_{1}, \ldots, t_{m}\right)$ whose coordinates satisfy the inequalities $0 \leqslant t_{i} \leqslant T_{i}$, where $T=\left(T_{1}, \ldots, T_{m}\right)$. Similarly, the interval $[0, \infty)$ means the set of points $t$ with $t_{i} \geqslant 0$. By the convolution

$$
\int_{0}^{t} g(t-\tau) k(\tau) d \tau
$$

we understand an integral stretched on the set $0 \leqslant \tau_{i} \leqslant t_{i}(i=1, \ldots, m)$. The proof of Theorem $I$ is based on the Titchmarsh theorem, which holds for any number of dimensions (see [5]). This theorem permits to introduce the class of $m$-dimensional operators $a=p / q$, where $q$ does not vanish identically in the $m$-dimensional right neighbourhood of 0 . Then all the preceding considerations remain true in the new, more general, interpretation.

\title{
References
}

[1] C. Foiaş, Approximation des opérateurs de J. Milcusiński par des fonctions continues, Studia Math. 21 (1961), p. 73 - 74.

[2] J. Mikusiński, Operational Oalculus, 1959.

[3] - An approximation Theorem and its applications in Operational Oal. culus, Studia Math. 27 (1966), p. 141-145.

[4] - Germs and their Operational Oaloulus, ibidem 26 (1966), p. 315-325.

[5] - Oonvolution of functions of several variables, ibidem 20 (1961), p. $253-259$.

[6] K. Skórnik, A theorem on approximation in the olass $L^{p}$, Bull. Ac. Pol. Sci. 14,4 (1966), p. 195-196.

Regu par la Rédaction le 3. 1. 1964

\section{On generalized topological divisors of zero in $m$-convex locally convex algebras}

by

\section{W. ŻELAZKO (Warszawa)}

By a topological algebra we mean in this paper a topological linear space together with an associative jointly continuous multiplication. An element $x$ of a topological algebra $A, x \neq 0$, will be called a left (right) topological divisor of zero if there exists a non-void subset $P \subset A$ such that zero is not in the closure $\bar{P}$ of $P$ but $0 \epsilon \overline{x P}(0 \epsilon \overline{P x})$. Here, as usual, $U V$ $=\{x y: x \in U, y \in V\}$. An element $x \in A$ is called a topological divisor of zero in $A$ if it is both a right and a left topological divisor of zero. It is a classical fact of the theory of Banach algebras, due to Šilov [3] (for algebras without a unit, see [5]) that a complex Banach algebra either possesses topological divisors of zero or is isomorphically homeomorphic to the field of complex numbers. The same holds for locally bounded algebras a class more general than the class of Banach algebras [5]. Here we in vestigate the problem for another generalization of Banach algebras, namely for the class of locally convex multiplicatively convex topological algebras (shortly, we shall call them m-convex algebras throughout this paper). An m-convex algebra is a topological algebra (over complexes) with a basis for neighbourhoods of the origin consisting of sets $\{U\}$ which are convex, symmetric and idempotent, i. e. such that $U U \subset U$. Or, which is equivalent, it is a locally convex algebra with the topology given by means of family $\mathscr{P}$ of submultiplicative pseudonorms:

$$
\|x y\| \leqslant\|x\|\|y\|
$$

and, in the case where the algebra in question possesses a unit $e$,

$$
\|e\|=1 .
$$

for each $\|\cdot\| \epsilon \mathscr{P}$. We may assume that $\mathscr{P}$ consists of all continuous pseudonorms satisfying (1) and (2) in the case where there is a unit element. The theory of these algebras was created by Arens [1] and Michael [2].

The statement that an $m$-convex algebra either possesses topological divisors of zero or is isomorphically homeomorphic to the field of 
complex numbers does not remain true: there are no topological divisors of zero in the algebra $E$ of all entire functions of one complex variable with pointwise addition and multiplication and with the compact-open topology (the topology of uniform convergence on compact subsets. of the complex plane). However, in this algebra there exist two sequences of entire functions such that the distances $\varrho\left(p_{n}, 0\right)>\delta>0, \varrho\left(\psi_{n}, 0\right)>\delta$ $>0, n=1,2, \ldots$ ( $E$ is clearly metrisable) but lim. $\varphi_{n} \psi_{n}=0$. That means that in $E$ there are sequential divisors of zero - a special case of the following general concept:

D ofinition. Let $A$ be a topological algebra. A pair of subsets $P, Q \subset A$ is called a pair of generalized topological divisors of zero in $A$ if $0 \phi \bar{P} \cup \bar{Q}$, but $0 \in \overline{P Q}$.

The concept of generalized topological divisors of zero was introdnced by the author in paper [4], where it was proved that a topological division algebra over complexes either possesses generalized topological divisors of zero or is isomorphically homeomorphic to the field of complex numbers.

In this paper we shall show that the above statement on division algebras holds true for the class of complex $m$-convex algebras, thus, for that class, we answer in the affirmative a general question posed in [4], and this is a generalization of the theorem of Silov mentioned above.

Without loss of generality we may limit ourselves to the commutative case, since it is sufficient to construct such divisors in any commu.. tative subalgebra of the algebra in question, and since any complex noncommutative algebra possesses a commutative subalgebra non-isomorphic with the complex numbers field. We may also limit ourselves to the case of complete $m$-convex algebras (cf. [2], section 5 ). In fact, any $m$-convex algebra is a dense subalgebra of a complete $m$-convex algebra. On the other hand, it is obvious that if a topological algebra with jointly continuous multiplication possesses generalized topological divisors of zero, then there are such divisors in any dense subalgebra.

We now recall some properties of (complete) commutative complex $m$-convex algebras, which shall be useful in the sequel (cf. [2] or. [4]).

If $A$ is an $m$-convex algebra with unit $e$, then there exists in $A$ at least one multiplicative and linear functional $f$, continuous in $A$. Denote by $\mathfrak{N}$ the set of all continuous multiplicative and linear functionals defined in $A$. An element $x \in A$ is invertible in $A$ if and only if $f(x) \neq 0$ for each $f \in \mathfrak{M}$. If $W_{n}(\lambda)$ is a sequence of polynomials of one complex variable $\lambda$, with complex coefficients, and if $W_{n}(\lambda)$ tend uniformly to zero on each compact subset of the complex plane, then for any $x \in A$, and \|\|$_{\epsilon} \mathscr{\mathscr { P }}$, $\lim \left\|W_{n}(x)\right\|=0$.

LEMMa 1. Let $\mathbb{E}$ be the m-convex algebra of all entire functions of one complex variable $\lambda$, with pointwise addition and multiplication and with the topology of uniform convergence on compact subsets of the complex plane; then $E$ possesses generalized topological divisors of zero.

Proof. Set $\tilde{\varphi}_{n}(\lambda)=(n+\lambda) /(n+1)$, and $\tilde{\psi}_{n}(\lambda)=(n-\lambda) /(n+1)$. For $|\lambda|<\sqrt{n}$ we have

$$
\left|\tilde{\varphi}_{n}(\lambda) \tilde{\psi}_{n}(\lambda)\right|=\frac{\left|n^{2}-\lambda^{2}\right|}{(n+1)^{2}} \leqslant \frac{n^{2}+n}{n^{2}+2 n+1}<1,
$$

and so for suitable integer $k_{n}$ we have

$$
\sup _{|\lambda|<\sqrt{n}}\left|\tilde{\varphi}_{n}(\lambda) \tilde{\psi}_{n}(\lambda)\right|^{k_{n}} \leqslant \frac{1}{n} .
$$

If we now put $\varphi_{n}(\lambda)=\left(\tilde{\varphi}_{n}(\lambda)\right)^{k_{n}}, \psi_{n}(\lambda)=\left(\tilde{\psi}_{n}(\lambda)\right)^{k_{n}}$, we have $\lim \varphi_{n} \psi_{n}=0$ in $E$. On the other hand, $\varphi_{n}(1)=\psi_{n}(-1)=1$, and since the functionals $F_{p}(\varphi)=\varphi(p)$ are continuous functionals in $A$, it follows that there is in $A$ a neighbourhood $U$ of the origin such that $\varphi_{n}, \psi_{n} \notin U, n=1,2$. Thus setting $P=\left\{\varphi_{n}\right\}, Q=\left\{\psi_{n}\right\}$, we have $0 \phi \bar{P} \cup \bar{Q}$, and $0 \in \overline{P Q}$, q.e.d.

LEMMA 2. If for a commutative m-convex algebra with unit e the set $\mathfrak{N}$ of continuous multiplicative and linear functionals consists of at least two elements, then A possesses generalized topological divisors of zero.

Proof. Let $f_{1}, f_{2} \in \mathfrak{M}, f_{1} \neq f_{2}$. There exists an $x \in A$ such that $\alpha=f_{1}(x) \neq \beta=f_{2}(x)$. If we set

$$
y=\frac{2}{\alpha-\beta}(x-\beta e)-e
$$

we have $f_{1}(y)=1$, and $f_{2}(y)=-1$. Now take polynomials $\varphi_{n}$ and $\psi_{n}$ constructed in the proof of Lemma 1. Since $\varphi_{n} \psi_{n}$ tends uniformly to zero on compact subsets of the complex plane, we have, for any $\|\cdot\| \in P$, $\lim \left\|\varphi_{n}(y) \psi_{n}(y)\right\|=0$. This implies $0 \epsilon \overline{\left\{\varphi_{n}(y)\right\}\left\{\psi_{n}(y)\right\}}$. On the other hand, $f_{1}\left(\varphi_{n}(y)\right)=\varphi_{n}\left(f_{1}(y)\right)=\varphi_{n}(1)=1$, similarly $f_{2}\left(\psi_{n}(y)\right)=1$; so, by the continuity of functionals $f_{1}$ and $f_{2}$, there exists in $A$ a neighbourhood $U$ of zero such that $\left[\left\{\varphi_{n}(y)\right\} \cup\left\{\psi_{n}(y)\right\}\right] \cap U=\emptyset$, and so $0 \notin \overline{\left\{\varphi_{n}(y)\right\}} \cup \overline{\left\{\psi_{n}(y)\right\}}$. Thus the sets $\left\{\varphi_{n}(y)\right\},\left\{\psi_{n}(y)\right\}$ form a pair of generalized topological divisors of zero in $A$, q.e.d.

LEMMA 3. If in a commutative and complete m-convex algebra $A$ with unit e there exists exactly one continuous multiplicative and linear functional $f$, then $A$ possesses generalized topological divisors of zero provided $A$ is not isomorphic to the field of complex numbers.

Proof. If $A$ is not the field of complexes, then there is an $x \in A$ such that $x \neq 0$, and $f(x)=0$. Put

$$
x_{n}=x+\frac{1}{n} e ;
$$

since $f\left(x_{n}\right)=1 / n \neq 0$, this is a sequence of invertible elements in $A$. 
We now fix a pseudonorm $\|\cdot\|_{0}$ in $\mathscr{P}$ such that $\|x\|_{0} \neq 0$; we may assume $\|x\|_{0}=2$. We may also assume that $\lim \left\|x_{n}^{-1}\right\|_{0}=\infty$. (If $\left\|\infty_{n}^{-1}\right\|$ was a bounded sequence for any $\|\cdot\| \epsilon P$, then $x$ would be an invertible element of $A$, the proof is exactly the same as in the case of Banach algebras, see e.g. [5].) We now put $y_{n}=x_{n}^{-1} /\left\|x_{n}^{-1}\right\|_{0}$. For any $\|\cdot\| \epsilon \mathscr{P}$ we have $\left\|x_{n} y_{n}\right\|=1 /\left\|x_{n}^{-1}\right\|_{0} \rightarrow 0$, and so $0 \epsilon \overline{\left(x_{n}\right)(y)_{n}}$. On the other hand, we have $\left\|y_{n}\right\|_{0}=1$, and $\left\|x_{n}\right\|_{0}>\|x\|_{0}-1 / n>1$, so $0 \notin \overline{\left(x_{n}\right)} \cup \overline{\left(y_{n}\right)}$ and the sets $\left(x_{n}\right)$, $\left(y_{n}\right)$ form a pair of generalized topological divisors of zero in A, q.e.d.

Lemma 2, Lemma 3 and the remark that any non-commutative $m$-convex algebra contains a non-zero commutative subalgebra not isomorphic to the field of complex numbers imply

Proposirion 1. Let $A$ be a complex m-convex algebra with a unit; then either $A$ possesses generalized topological divisors of zero, or $A$ is isomorphically homeomorphic to the field of complex numbers.

Remark 1. In complete algebras the divisors of zero may be chosen in the form of two sequences $\left(x_{n}\right),\left(y_{n}\right) \subset A$ such that for each $\|\cdot\| \epsilon \mathscr{P}$ we bave $\lim \left\|x_{n} y_{n}\right\|=0$, and for some $\|\cdot\|_{0} \epsilon \mathscr{P}$ we have $\left\|x_{n}\right\|_{0}=\left\|y_{n}\right\|_{0}=1$. (In Lemma 2 as the pseudonorm $\|x\|_{0}$ we may take $\|x\|_{0}=\max \left\{\left|f_{1}(x)\right|\right.$, $\left.\left|f_{2}(x)\right|\right\}$.)

To prove a similar result on algebras without a unit we need the following

LEMma 4. Let $A$ be an m-convex algebra without generalized topological divisors of zero; then for each $|\cdot| \epsilon \mathscr{P}$ there exists a pseudonorm $\|\cdot\|$ in $\mathscr{P}$ such that for some $C>0$

$$
\|x y\| \geqslant C|x||y| \text { for each } x, y \in A .
$$

Proof. If (3) does not hold, then there exists a pseudonorm $|\cdot| \epsilon \mathscr{P}$ such that for each $\|\cdot\| \epsilon \mathscr{P}$ and each natural numiber $k$ there exist elements $x_{k}, y_{k} \in A$ such that

$$
\left\|x_{k} y_{k}\right\|<\frac{1}{k}\left|x_{k}\right|\left|y_{k}\right| .
$$

We may assume $\left|x_{k}\right|=\left|y_{k}\right|=1$. Setting $P=\{x \in A:|x|=1\}$, we have $0 \notin \bar{P}$. On the other hand, for an arbitrary neighbourhood $U$ of the origin there is a pseudonorm $\|\cdot\| \epsilon \mathscr{P}$ and an integer $\%$ such that $\{x \in A$ : $\|x\|<1 / k\} \subset U$. Taking, by (4), suitable $x_{k}, y_{k}$ for the pseudonorm $\|\cdot\|$, we have $x_{k} y_{k} \in U$. Therefore $0 \epsilon \overline{P^{2}}$, and there are in $A$ generalized topological divisors of zero. This is a contradiction which proves formula (3).

Remark 2. Since $\|\cdot\|$ in formula (3) is a submultiplicative pseudonorm, it follows that $|\cdot|$ is continuous with respect to $\|\cdot\|$ and there exists a positive constant $K$ such that

$$
|x| \leqslant K\|x\| .
$$

Proposition 2. Let $A$ be a complete (commutative) complex m-convex algebra without a unit element; then $A$ possesses generalized topological divisors of zero.

Proof. Define an $m$-convex algebra $\tilde{A}$ consisting of all formal sums $x+\lambda e, x \in A, \lambda-a$ complex scalar, with multiplication defined by

$$
\left(x_{1}+\lambda_{1} e\right)\left(x_{2}+\lambda_{2} e\right)=x_{1} x_{2}+\lambda_{1} x_{2}+\lambda_{2} x_{1}+\lambda_{1} \lambda_{2} e,
$$

and pseudonorms defined by

$$
\|x+\lambda e\|=\|x\|+|\lambda|, \quad\|\cdot\| \epsilon \mathscr{P} .
$$

$\tilde{A}$ is a complete $m$-convex algebra with unit $e$, and so by Proposition 1 and Remark 1 there exist sequences $\left(x_{n}+\lambda_{n}^{1} e\right),\left(y_{n}+\lambda_{n}^{2} e\right) \subset \tilde{A}$, such that

$$
\lim \left\|\left(x_{n}+\lambda_{n}^{1} e\right)\left(y_{n}+\lambda_{n}^{2} e\right)\right\|=0 \quad \text { for each }\|\cdot\| \epsilon P,
$$

and for some $\|\cdot\|_{0} \in \mathscr{P}$ we have

$$
\left\|x_{n}+\lambda_{n}^{1} e\right\|_{0}=1=\left\|y_{n}+\lambda_{n}^{2} e\right\| .
$$

It follows that the sequences $\lambda_{n}^{1}, \lambda_{n}^{2}$ are bounded; thus, by passing, if necessary, to a subsequence we may assume that there exist limits $\lambda^{1}=\lim \lambda_{n}^{1}$, and $\lambda^{2}=\lim \lambda_{n}^{2}$. By $(6)$ we have either $\lambda^{1}=0$ or $\lambda^{2}=0$, and so by symmetry we may assume $\lambda^{1}=0$. We may then rewrite $(6)$ in the form

$$
\lim \left\|x_{n} y_{i_{2}}+\lambda_{n}^{1} y_{n}+\lambda_{n}^{2} x_{n}\right\|=0 \quad \text { for each }\|\cdot\| \epsilon \mathscr{P} .
$$

By (7) we have $\left\|x_{n}\right\|_{0} \rightarrow 1$. Let us remark that we cannot have $\lim \left\|y_{n}\right\|=0$ for each $\|\cdot\| \epsilon \mathscr{P}$, since in that case, by (8), it we would have $\lim \left\|x_{n} y_{n}+\lambda_{n}^{2} x_{n}\right\|=0$, and so $\lim \left|\left\|x_{n} y_{n}\right\|-\right| \lambda_{n}^{2}\left|\left\|x_{n}\right\|\right|=0$, which is impossible, because $\lim \left|\lambda_{n}^{2}\right|=1$, and $\left\|x_{n} y_{n}\right\| \leqslant\left\|x_{n}\right\|\left\|y_{n}\right\|$. We may assume, therefore, that

$$
\left\|x_{n}\right\|_{0}>\delta>0, \quad\left\|y_{n}\right\|_{0}>\delta>0
$$

taking, if necessary, some bigger norm instead of $\|\cdot\|_{0}$.

We shall now discuss a number of possible situations. Each time we may assume that there are no generalized topological divisors of zero, and so, by applying Lemma 4 try to arrive at a contradiction.

$1^{\circ}$ The sequences $\left(x_{n}\right),\left(y_{n}\right)$ are both unbounded in $A$. In this case we may assume that there exists an element $|\cdot| \epsilon \mathscr{P}$ such that

$$
\lim \left|x_{n}\right|=\lim \left|y_{n}\right|=\infty,
$$


by passing, if necessary to a subsequence (otherwise, by passing to a subsequence, we would find that one of the sequences is bounded in $A$ ). Let $\|\cdot\|$ denote a continuous submultiplicative pseudonorm corresponding to $|\cdot|$ by formula (3). By Lemma 4, and Remark 2 we have

$$
\left\|x_{n} y_{n}+\lambda_{n}^{1} y_{n}+\lambda_{n}^{2} x_{n}\right\| \geqslant O\left|x_{n}\right|\left|y_{n}\right|-K\left|\lambda_{n}^{1}\right|\left|y_{n}\right|-K\left|\lambda_{n}^{2}\right|\left|x_{n}\right|
$$

The right-hand term of (11) tends, by (10), to infinity, while the left-hand term tends, by (8), to zero, which gives a contradiction, and shows that in this ease there are generalized topological divisors of zero in $A$.

$2^{\circ}$ Suppose that the sequence $\left(x_{n}\right)$ is bounded, while $\left(y_{n}\right)$ is an unbounded sequence. There exists a sequence $\alpha_{n}$ of scalars, $\alpha_{n} \rightarrow 0$, such that $a_{n} y_{n}$ remains unbounded. Thus, multiplying the $n$-th term in formula (8) by $\alpha_{n}$, and setting $z_{n}=\alpha_{n} y_{n}$, we may rewrite (8) as

$$
\lim \left\|x_{n} z_{n}+\lambda_{n}^{1} z_{n}\right\|=0 \quad \text { for each }\|\cdot\| \epsilon \mathscr{P} .
$$

We can find such a pseudonorm $|\cdot| \epsilon P$ that $\lim \left|z_{n}\right|=\infty$ (passing, if necessary, to a subsequence). If we assume that there are no generalized topological divisors of zero in $A$, we may find, by formula (3) of Lemma 4, a pseudonorm $\|\cdot\|$ corresponding to $|x|_{1}=\max \left\{\|x\|_{0},|x|\right\}$. We have

$$
|| x_{n} z_{n}+\lambda_{n}^{1} z_{n} \| \geqslant C\left|x_{n}\right|_{1}\left|z_{n}\right|_{1}-K\left|\lambda_{n}^{1}\right|\left|z_{n}\right|_{1}
$$

which gives a contradiction, since the left-hand term tends, by (12), to zero, while the right-hand term tends, by (9), to infinity. Thus in this case the proposition is proved.

$3^{\circ}$ Suppose that also $\lambda^{2}=0$. If $\left(a_{n}\right),\left(y_{n}\right)$ are botb bounded, then (8) reads as

$$
\lim \left\|x_{n} y_{n}\right\|=0 \quad \text { for each }\|\cdot\| \epsilon \mathscr{P},
$$

and, by relations (9), the proposition is proved. If $\left(x_{n}\right)$ is an unbounded sequence, while $\left(y_{n}\right)$ is bounded, then (8) reads as

$$
\lim \left\|x_{n} y_{n}+\lambda_{n}^{2} x_{n}\right\|=0 \quad \text { for each }\|\cdot\| \epsilon \mathscr{P},
$$

and this is exactly, by formula (12), the situation of section $2^{\circ}$. So in this case our proposition is also proved.

We have to consider the remaining case, in which $\lambda^{2} \neq 0,\left(x_{n}\right)$ is unbounded, while $\left(y_{n}\right)$ is a bounded sequence. In this case (8) reads as

$$
\lim \left\|x_{n} y_{n}+\lambda_{n}^{2} x_{n}\right\|=0, \ldots\|\| \epsilon \mathscr{P} ;
$$

so, substituting $y_{n}$ by $-y_{n} / \lambda_{n}^{2}$, we infer from this formula that

$$
\lim \left\|x_{n} y_{n}-x_{n}\right\|=0 \quad \text { for each }\|\cdot\| \epsilon P,
$$

while (9) hold true. Now consider the following situations:

$4^{\circ}$ There exists a $z \in A$ such that for some $\|\cdot\|_{0} \in \mathscr{P}$

$$
\overline{\lim }\left\|z y_{n}-z\right\|_{0} \neq 0 \text {. }
$$

In this case, setting $z_{n}=z y_{n}-z$ and passing, if necessary, to a subsequence, we see that, for some $\delta>0,\left\|z_{n}\right\|_{0}>\delta$, while

$$
\lim \left\|x_{n} z_{n}\right\|=0 \quad \text { for each }\|\cdot\| \epsilon \mathscr{P} ;
$$

thus, in this case, there are generalized topological divisors of zero in $A$.

$$
5^{\circ} \text { Suppose that }
$$

$$
\lim \left\|z y_{n}-z\right\|=0 \quad \text { for each }\|\cdot\| \epsilon \mathscr{P}, z \in A,
$$

but $y_{n}$ is not a Cauchy sequence with respect to any $\|\cdot\| \epsilon P$. There exists, therefore, a pseudonorm $\|\cdot\|_{\odot} \epsilon \mathscr{P}$, such that for a certain sequence of integers $\left(k_{n}\right)$, and some $\delta>0$ we have $\left\|y_{n}-y_{k_{n}}\right\|_{0}>\delta$. On the other hand, for any $z \in A$ we have $\lim \left\|z\left(y_{n}-y_{k_{n}}\right)\right\|=0$, for each $\|\cdot\| \epsilon \mathscr{P}$, and so any non-zero element of $A$ is a topological divisor of zero.

$6^{\circ}$ Suppose that (14) holds, and $\left(y_{n}\right)$ is a Cauchy sequence with respect to each pseudonorm belonging to $\mathscr{P}$.

In this case the completeness of $A$ implies that there exists an element $y \in A$, such that for any $\|\cdot\| \epsilon \mathscr{P}$ we have $\lim \left\|y_{n}-y\right\|=0$. From (14) it follows that $y$ is a unit element in $A$, which contradicts the assumption that $A$ possesses no unit. This completes the proof of Proposition 2.

From Propositions 1 and 2 , and from the remarks that any $m$-convex algebra is a dense subalgebra of a complete $m$-convex algebra and any dense subalgebra of an algebra possessing generalized topological divisors of zero also possesses such divisors follows

Theorem 1. Let $A$ be a complex $\left(^{1}\right)$ m-convex algebra. Then either $A$ possesses generalized topological divisors of zero or $A$ is isomorphically homeomorphic to the field of complex numbers.

\section{References}

[1] R. Arens, A generalization of normed rings, Pacific J. Math. 2 (1952), p. $455-471$.

[2] E. Michael, Locally multiplicatively-convex topological algebras, Mem. Amer. Math. Soc. 11 (1952).

\footnotetext{
(1) An analogous result has been obtained for real algebras [6] (added in proof).
} 
[3] Г. Е. Шилов, О расиирении максимальлых идеалов, Докл. АН СССР 29 (1940), p. 83-85.

[4] W. Zelazko, Metric generalizations of Banach algebras, Rozprawy Matematyczne 47 (1965).

[5] - A note on topological divisors of zero in p-normed algebras, Coll. Math (in print).

[6] - On generatized topological divisors of zero in real m-convex algebras, Studia Math. (to appear).

INSTITUTE OF MATHEMATICS OF THE POMISH ACADHMY OF SCIENCES INSTYTUT MATEMATYCZNY POLSKIEJ AKADEMII NAUK

Reşu par la Rédaction le 20.11. 1965

\section{A generalized function calculus based on the Laplace transform*}

by

CHARLES SWARTZ (Now Mexico, U. S. A.)

\section{Introduction}

In this paper the classical Laplace transform is extended in a very natural way to a space of generalized functions. This extension is carried out by utilizing a method for constructing generalized functions suggested by Mikusiński [5]. This method has been used to construct Schwartz's space $D^{\prime}[8]$ and to extend the Fourier transform to a space of generalized functions [4].

The usual operations of translation, addition, etc. are defined for the g.f.'s (g.f. = generalized function), and the classical formulas pertaining to such operations are extended to the g.f.'s. Differentiation, integration, and convergence are defined and the usual limit interchanges in distribution theory are justified. In particular, the convergence defined is the "weak" sequential convergence suggested by Mikusiński, and it is shown that the g. f. space is "complete" with respect to this sequential convergence. Using this completeness property, it is shown that the Laplace transform maps the $g$. $f$. space onto the class of all functions which are analytic in some half plane $\mathrm{R} z>a \geqslant 0$. We also give several characterizations of the g.f.'s which are distributional derivatives of continuous functions of exponential order, and an inversion formula for such g. f.'s is presented.

Convolution is defined for the g.f.'s, and we give conditions under which the convolution equation $A * X=B$ has a solution $X$. Multiplication of a g. f. by a suitably well-bebaved function is also defined. In the final section, we consider g. f.'s depending on a parameter and establish some of the limit interchanges that have been used formally in the operational calculus.

\section{Preliminaries}

We will denote by $A$ the class of all functions $f(z)$ which are analytic in some half plane $\mathrm{R} z>a \geqslant 0$. The half plane may depend on the function. A sequence $\left\{f_{n}(z)\right\}$ of functions in $A$ converges to $f(z)$ in $A$ if there exists

* This paper is based on my doctoral dissertation presented to the University of Arizona. 